\title{
Belphégor
}

Littérature populaire et culture médiatique

$17 \mid 2019$

Mutations des légitimités dans les productions culturelles contemporaines

\section{Les apprenants du MOOC fantasy de l'université d'Artois : réception et retransmission des savoirs}

Marie Lucie Bougon

\section{(2) OpenEdition}

Journals

Electronic version

URL: https://journals.openedition.org/belphegor/1899

DOI: 10.4000/belphegor.1899

ISSN: 1499-7185

Publisher

LPCM

Electronic reference

Marie Lucie Bougon, "Les apprenants du MOOC fantasy de l'université d'Artois : réception et retransmission des savoirs", Belphégor [Online], 17 | 2019, Online since 18 April 2019, connection on 25 August 2021. URL: http://journals.openedition.org/belphegor/1899 ; DOI: https://doi.org/10.4000/ belphegor.1899

This text was automatically generated on 25 August 2021.

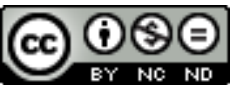

Belphégor est mis à disposition selon les termes de la Licence Creative Commons Attribution - Pas d'Utilisation Commerciale - Pas de Modification 4.0 International. 


\title{
Les apprenants du MOOC fantasy de l'université d'Artois : réception et retransmission des savoirs
}

\author{
Marie Lucie Bougon
}

1 Créé en 2015 par l'université d'Artois, le MOOC « Fantasy, de l'Angleterre victorienne au Trône de fer » se présente de la façon suivante sur FUN : «Ce MOOC sera consacré au genre littéraire et médiatique de la fantasy. Il permettra d'approfondir un goût aujourd'hui largement partagé grâce à de nombreuses œuvres à succès (Harry Potter, Le Seigneur des Anneaux, Game of Thrones...). On y présentera l'ampleur et la diversité thématique et historique de la fantasy : influence des mythes, héritage du Moyen Âge, grands auteurs du XIX ${ }^{\mathrm{e}}$ au XXI ${ }^{\mathrm{e}}$ siècle, romans, films ou jeux visant les enfants, les adolescents ou les adultes. Les cours seront assurés par une équipe de spécialistes du domaine. $»^{1}$

2 Le format du MOOC est également énoncé de façon très claire : il se compose de cours magistraux filmés, disponibles au format vidéo, audio, et texte (en pdf), il se divise en plusieurs modules qui peuvent être validés via des exercices tels que des QCM, et la validation finale s'effectue grâce à un travail rédigé, puis corrigé par les pairs. Il s'agit donc d'un MOOC qui alterne entre une pratique individuelle, solitaire (visionnage des cours, QCM) et une pratique collective (évaluation par les pairs.) Le cours est explicitement présenté comme s'adressant au grand public.

3 La première édition du MOOC fantasy, en 2015, a attiré 9296 inscrits. La seconde en 2016, en a compté 6354, et la troisième et dernière, en 2017, en a rassemblé 6529 . On observe donc une baisse assez importante entre la première et la seconde édition, puis une hausse légère lors de la troisième, sachant que certains apprenants se sont réinscrits chaque année.

Lors de ces trois éditions, le forum du MOOC a été extrêmement actif, et les milliers de messages échangés entre les utilisateurs constituent un corpus très riche. Que nous apprennent-ils donc sur les modalités d'échanges de savoirs horizontaux sur un domaine lui-même encore assez peu « légitime », la fantasy? 
$5 \quad$ Notre étude s'appuie sur l'analyse des fils de discussion du forum ainsi que sur les productions collaboratives et les informations de profil des apprenants (sachant que de nombreux profils sont malheureusement vierges). Nous avons recensé et étudié ces données principalement sans aide logicielle - à l'exception de quelques utilisations occasionnelles de Tropes $^{2}$, qui seront signalées au fil de l'article. Nous avons travaillé sur les trois éditions du MOOC, ce qui implique, par moments, des disparités entre les données disponibles selon les années : les nuages de mots et les sondages effectués auprès des apprenants ne sont apparus sur FUN qu'au cours de la troisième édition.

6 Nous nous pencherons, dans un premier temps, sur les informations renseignées par les apprenants dans leurs profils virtuels, puis, nous nous interrogerons sur la réception du MOOC et la fertilité des échanges entre pairs en analysant les fils de discussion du forum, et enfin, nous étudierons le dépassement du rapport de transmission vertical en nous intéressant à la retransmission horizontale et aux travaux collaboratifs des apprenants.

\section{Apprenants du MOOC : quels profils?}

7 De la disputatio médiévale au compagnonnage ou aux cercles épistémiques, les manières d'apprendre ensemble ont toujours abondé, et les MOOC n'en sont que les manifestations les plus contemporaines. Si la formation à distance par voie postale existe depuis le XVIII ${ }^{\mathrm{e}}$ siècle ${ }^{3}$, l'avènement d'Internet a permis de réconcilier cette forme d'apprentissage non-présentiel avec une pratique collective et de rompre l'isolement souvent inhérent à la formation à distance. Les formes contemporaines de l'e-learning se déclinent en plusieurs variantes. Les OER, ou Open Educational Resources, correspondent à l'ensemble des ressources accessibles gratuitement en ligne, qu'il s'agisse de cours ou de conférences enregistrées, de vidéos pédagogiques sur Youtube, de podcasts, de documents numérisés ou de logiciels en open source. Les MOOC (Massive Open Online Course), quant à eux, ont une définition plus restreinte : « Un MOOC, c'est un cours magistral en ligne, ou plutôt un cortège de clips magistraux n'excédant pas dix à quinze minutes chacun, agrémenté d'un forum organisant l'interaction entre les usagers et parfois assorti d'un réseau social encadrant l'évaluation par les pairs. Un MOOC peut ou non donner lieu à un certificat d'achèvement, voire à l'attribution de crédits en vue d'un diplôme. $»^{4}$ La plupart des MOOC sont gratuits, mais ce n'est pas une loi absolue : Thierry Karsenti rappelle justement que Georgia Tech University, par exemple, a déjà proposé des $\mathrm{MOOC}$ diplômants à hauteur de 7000 dollars ${ }^{5}$. Il existe enfin les SPOC (Small Private Online Classes), plus confidentiels, créés afin de combler certaines lacunes des MOOC (notamment en termes de taux de complétion').

8 La catégorie qui nous intéresse ici, celle des MOOC, apparait dans les années 2010, et connaît un grand mouvement d'enthousiasme quand Sebastian Thrun, de l'université de Stanford, crée en 2011 un MOOC sur l'intelligence artificielle et recueille quasiment deux cent mille inscriptions. Dès lors, les MOOC se multiplient via les plateformes américaines Udacity, Coursera et EdX. Malheureusement, l'enthousiasme pour ceux-ci retombe rapidement (dès 2012), quand la plupart des organismes constatent qu'en dépit du très grand nombre d'inscrits, peu d'apprenants suivent le MOOC jusqu'au bout et obtiennent le certificat.

9 En France, le développement de la plateforme FUN est lancé en 2012 sous l'impulsion de Geneviève Fioraso, ministre de l'enseignement supérieur et de la recherche. La 
plateforme est active et propose des MOOC depuis 2014. Avant cela, seulement $3 \%$ des universités françaises proposaient des cours en ligne (contre $80 \%$ des universités américaines). Le ministère investit 12 millions d'euros dans le développement de FUN, ce qui reste assez bas en comparaison des budgets américains. L'infrastructure de FUN est rapidement critiquée, puisque la plateforme choisit de s'appuyer sur la technologie Open EdX, version open source de la plateforme américaine EdX, elle-même proposée par Google. Les données sont en revanche hébergées par l'INRIA, qui est un institut français. Le lancement de FUN peut être considéré comme un succès, puisque la plateforme compte aujourd'hui 150 MOOC et plus d'un million d'inscrits, qu'on appelle les « apprenants ».

Le néologisme "apprenance » et son dérivé "apprenant » sont issus des sciences de l'éducation : c'est Philippe Carré qui le crée en 1997. Il le définit comme « un ensemble durable de dispositions favorables à l'action d'apprendre dans toutes les situations formelles ou informelles, de façon expériencielle ou didactique, autodirigée ou non, intentionnelle ou fortuite. ${ }^{7}$ Il s'agit donc d'une façon plurielle d'apprendre, qui peut sortir du schéma transmissif traditionnel fondé sur le rapport vertical maître-élève. On comprend aisément pourquoi ce terme a gagné le domaine des MOOC, où il s'est cependant éloigné de son sens originel, puisqu'il est désormais généralement utilisé pour désigner l'ensemble des inscrits, que ceux-ci suivent ou non la totalité du cours (ce qui ne correspond pas tout à fait aux "conditions durables" mentionnées par Philippe Carré).

11 Nous avons choisi de nous focaliser dans cette première partie sur les informations tirées des profils virtuels des apprenants du MOOC, sachant que celles-ci sont parfois lacunaires. En effet, le forum du MOOC fantasy est plutôt minimaliste et ne permet pas aux inscrits de " personnaliser » leur identité virtuelle avec des citations, bannières ou avatars, il ne nous est donc pas possible d'effectuer une analyse aussi poussée que celle proposée par Anne-Sophie Béliard, par exemple, dans son étude d'un forum de fans de la série Prison Break ${ }^{8}$. Nous disposons ici d'une palette de données plus réduite, les informations remplies par les apprenants lors de leur inscription au MOOC, qui comprennent seulement six rubriques : genre, année de naissance, niveau d'éducation, ville, pays, objectifs. Il convient également de préciser qu'un nombre important d'apprenants a laissé certaines de ces rubriques vides : lors de la deuxième édition du MOOC, pour lequel nous comptons 5701 profils enregistrés, 522 apprenants n'ont pas renseigné leur année de naissance, 489 n'ont pas indiqué leur genre, 528 n'ont pas mentionné leur niveau d'étude, 891 n'ont pas précisé leur pays, et 2382 ont laissé la rubrique « objectifs » vierge. ${ }^{9}$ C'est donc avec prudence qu'il faut considérer les chiffres dont nous allons faire état dans cette première partie. Ces lacunes rendent également difficiles une analyse des interactions entre apprenants selon leur genre, leur niveau d'étude ou leur capital culturel.

D'après Denis Cristol, les apprenants des MOOC seraient généralement des "jeunes actifs de 30 à 40 ans déjà très diplômés. " ${ }^{10}$ Cette affirmation ne se vérifie pas tout à fait concernant la première édition du MOOC fantasy, qui a attiré un public plutôt jeune : un quart des participants a moins de 25 ans, la moitié d'entre eux a moins de 32 ans, les trois quarts d'entre eux ont moins de 42 ans. Ce public a légèrement «vieilli » lors des deux éditions suivantes : on voit que les plus de 40 ans sont majoritaires, suivis de près par la tranche d'âge 30-40 ans, ce qui se rapproche plus des chiffres mentionnés par Denis Cristol. (Figure 1) 


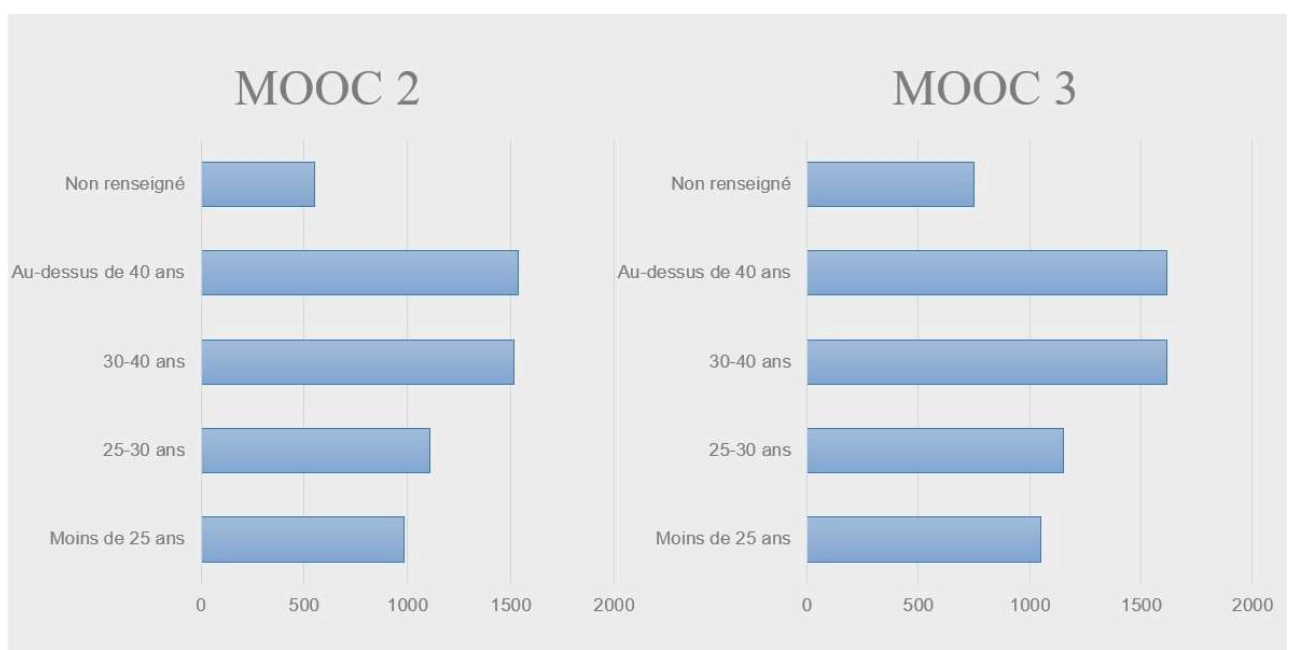

Figure 1 : évolution des âges des participants lors de la deuxième et de la troisième édition du MOOC fantasy

Concernant le genre des participants, on observe dans les trois MOOC une majorité importante de femmes (toujours plus de 60\%.) (Figure 2)

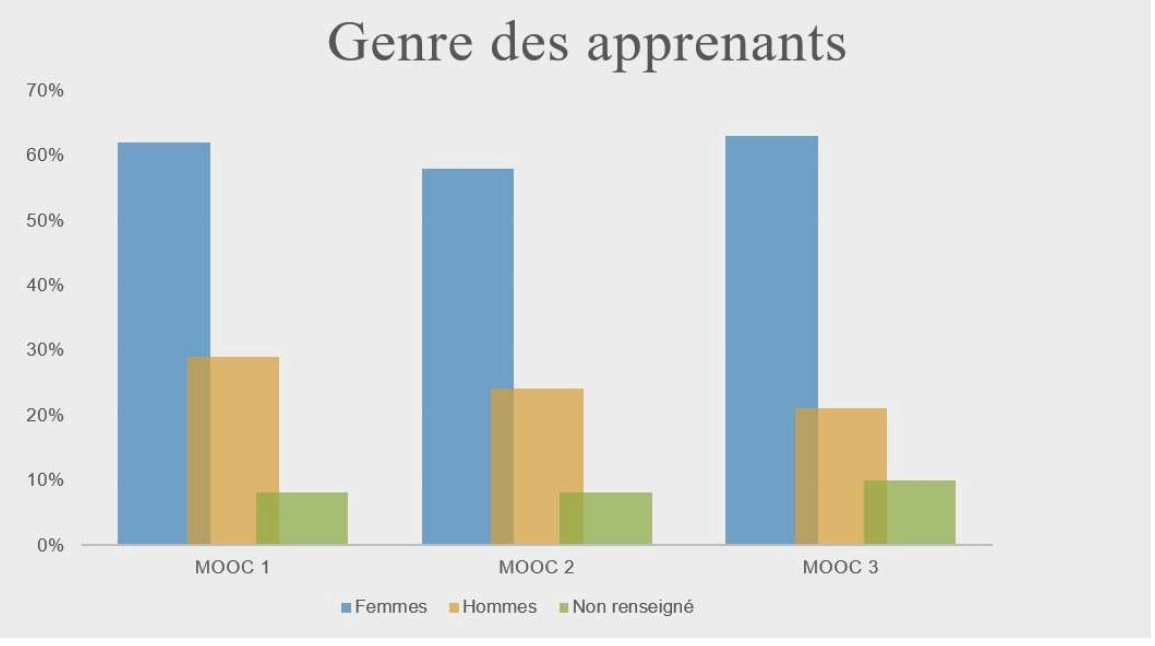

Figure 2 : genre des participants du MOOC fantasy

Le MOOC fantasy a principalement touché un public français : en étudiant la répartition géographique des apprenants de la troisième édition, on constate que $78 \%$ des candidats sont situés en France, $4 \%$ en Belgique, 1\% au Canada, 1\% en Suisse,1\% au Maroc, et $7 \%$ dans d'autres pays du monde ayant un nombre d'inscrits trop faibles pour qu'il soit nécessaire de créer une catégorie. On remarque également que dans les pays non-francophones, les apprenants du MOOC sont souvent des expatriés et le précisent dans la courte description de leur profil. (Figure 3) 


\section{Répartition géographique} (MOOC 3)

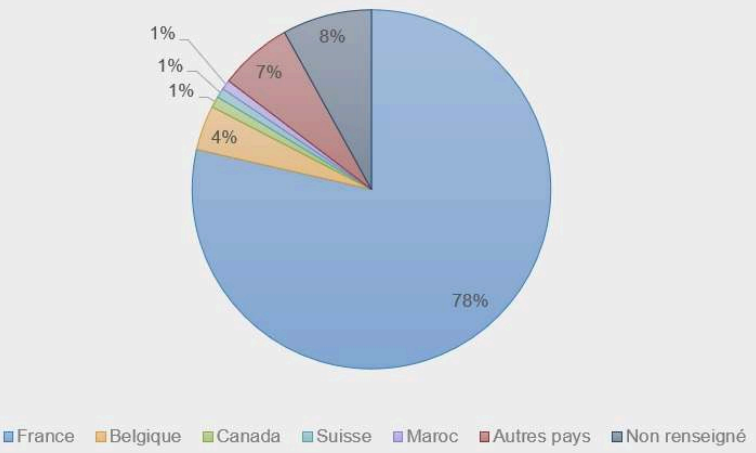

Figure 3 : répartition géographique des apprenants de la troisième édition du MOOC fantasy.

En ce qui concerne le niveau d'études, nous obtenons des résultats assez proches de ceux annoncés par Denis Cristol, puisqu'une majorité des apprenants de la première édition du MOOC est diplômée d'un master et que plus de la moitié d'entre eux a au moins une licence. (Figure 4)

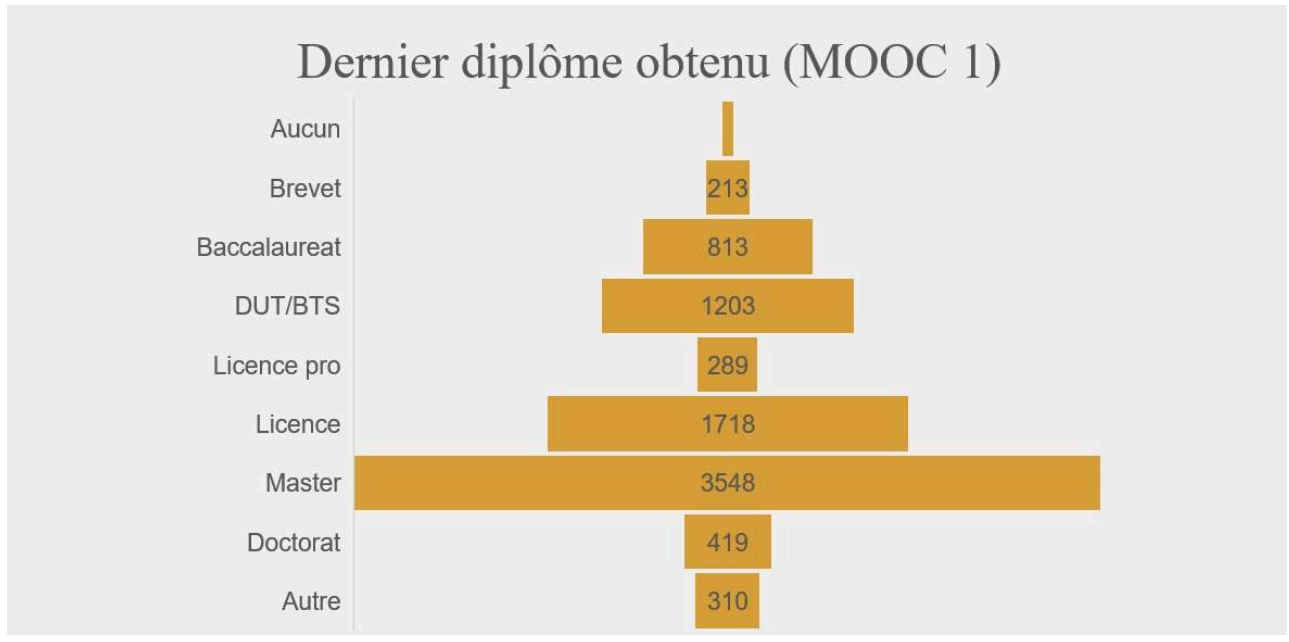

Figure 4 : Niveau d'études des apprenants de la première édition du MOOC fantasy

Nous avons également appris lors de la troisième édition du MOOC que les apprenants étaient majoritairement des lecteurs, puisque à la question « par quel média avez-vous découvert le monde de la fantasy?», 66\% de ceux qui ont accepté de faire le questionnaire répondent «par les romans. » Ce résultat est parfaitement cohérent avec la majorité observée de femmes parmi les apprenants, puisque l'enquête sur les pratiques culturelles des Français organisée par le ministère de la culture en 2008 indique que ce sont les femmes qui lisent le plus parmi la population française. ${ }^{11}$

Les préférences de lecture des apprenants peuvent être considérées comme « classiques » dans le domaine de la fantasy : d'après le nuage de mots réalisé au cours de la troisième édition, l'auteur favori entre tous reste Tolkien, suivi par Robin Hobb. (Figure 5) 


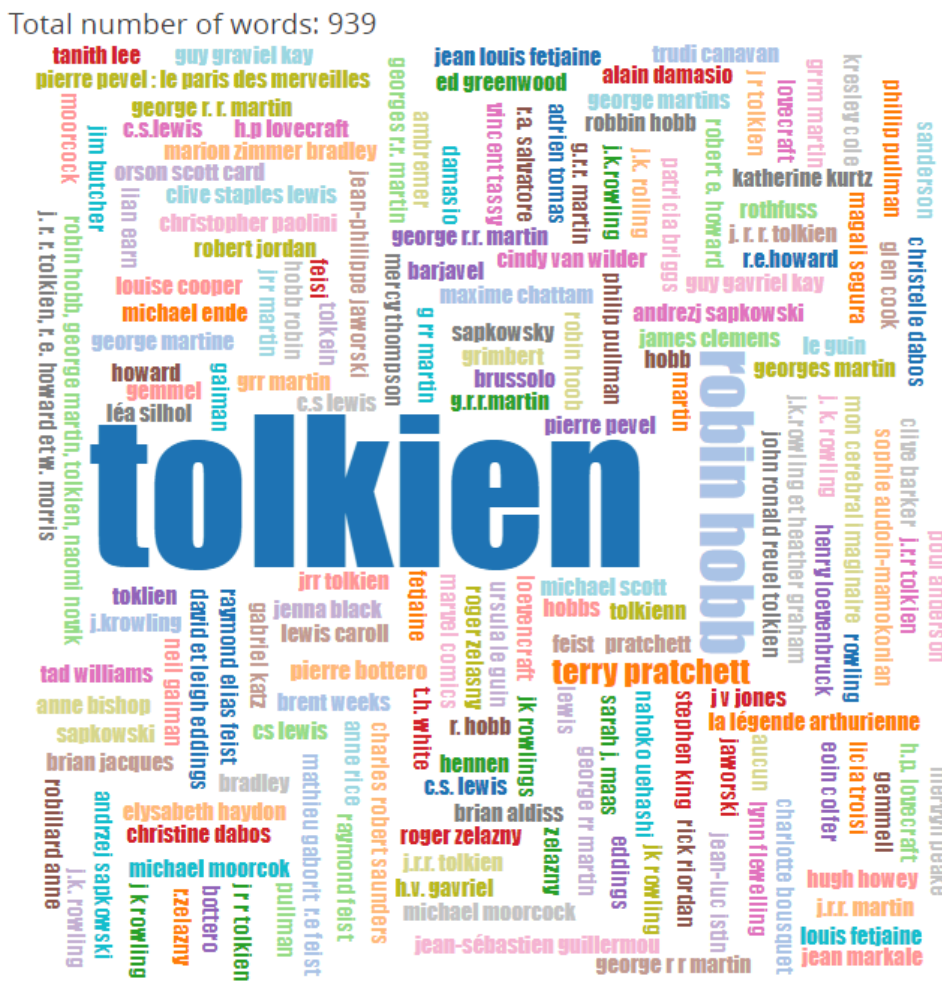

Figure 5 : Nuage de mots généré lors de la troisième édition du $\mathrm{MOOC}$ fantasy à partir des réponses à la question : « quel est votre auteur favori ? » sur FUN

18 Mais ces lecteurs de fantasy n'ont pourtant pas négligé le développement d'une culture plus académique: à la question «avez-vous déjà lu des textes médiévaux?»,40\% d'entre eux indiquent avoir lu plusieurs textes.

Ces constats sont assez cohérents avec les informations que nous avons pu relever en effectuant des recherches par mots-clefs dans les fichiers rassemblant les informations des profils des apprenants de la deuxième édition du MOOC : nous avons ainsi remarqué que parmi les 3319 apprenants ayant rempli la rubrique "objectifs" de leurs informations de profil, 81 sont professeurs ou souhaitent le devenir, 48 sont bibliothécaires, 31 documentalistes, et 11 libraires. Certains inscrits au MOOC exercent donc une profession en rapport avec le monde de l'enseignement ou avec celui du livre. Certains de ces mêmes apprenants ont d'ailleurs des attentes directement liées à leur pratique professionnelle. Nous relevons ainsi, toujours dans le MOOC 2, ces quelques citations (parmi de bien plus nombreuses sur le sujet) : «C'est un genre que je connais mal et dont je ne suis pas fan. Il s'agit plutôt pour moi de me repérer (sources mythologiques, matière médiévale, procédés...) et peut-être de m'y intéresser ... c'est l'univers de beaucoup d'élèves (je suis enseignante) » ou encore « Je suis enseignante en lycée professionnel et j'ai travaillé sur la série Le Trône de fer et je souhaiterais approfondir mes connaissances en littérature Fantasy afin d'offrir des cours de qualité à mes élèves. »

Le MOOC Fantasy semble, pour ces apprenants professeurs, devenir une sorte de formation continue venant remplacer ou compléter les formations proposées par l'Éducation Nationale. De même pour les bibliothécaires, qui ont ouvert sur les forums plusieurs fils de discussion afin de demander de l'aide pour classer les ouvrages de fantasy en rayon de façon juste et efficace. 

apprenants suivent le MOOC jusqu'au bout et soient en mesure d'obtenir leur attestation. En effet, Nicolas Oliveri nous indique que «environ seulement $5 \%$ des apprenants inscrits à un $\mathrm{MOOC}$, le fréquentent jusqu'à son terme et peuvent alors se targuer d'obtenir le certificat attestant du nombre d'heures obligatoires passées en ligne. » ${ }^{12}$, mais comme on peut l'observer sur ce graphique, le taux d'apprenants du MOOC fantasy pouvant générer une attestation oscille, selon les années, entre 14 et $17 \%$, ce qui est largement supérieur à la moyenne. (Figure 6)
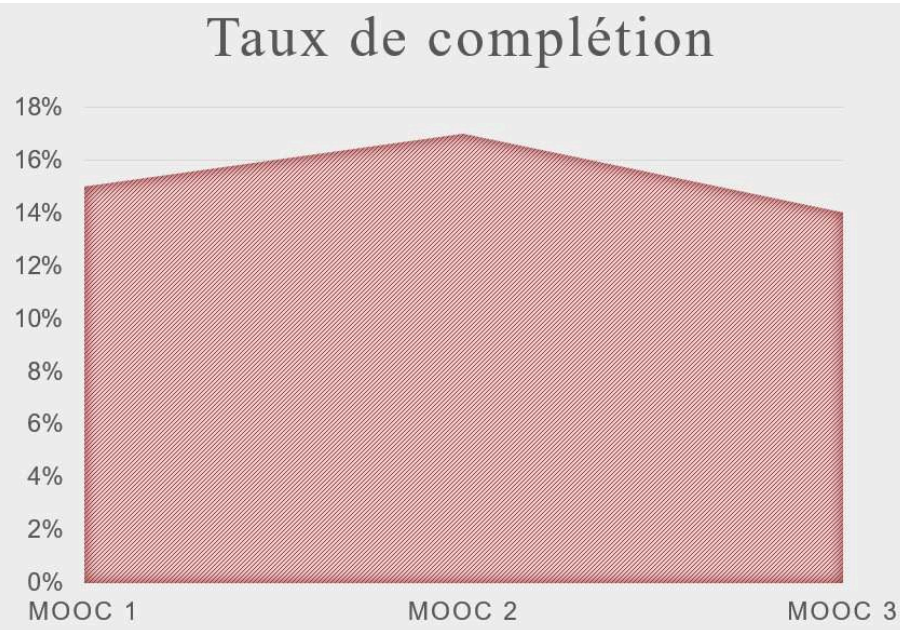

Figure 6 : Taux de complétion lors des trois éditions du MOOC fantasy

\section{Réception du MOOC et échanges entre pairs}

Un des premiers sujets abordés par les apprenants du MOOC est leur réception de celuici : ce qu'ils ont apprécié, ce qu'ils n'ont pas bien compris, ce qu'ils aimeraient encore approfondir, etc.

C'est lors de la première édition du MOOC que ces réactions ont été les plus marquées. Nous pouvons notamment y observer l'ouverture de topics spontanés afin d'exprimer une opinion sur le MOOC, comme, par un exemple, un fil intitulé "Un grand bravo", créé par DylanM à l'issue de la semaine 5, et ayant recueilli une quarantaine de réponses. Ici, les retours sont extrêmement positifs : en analysant le fil via le logiciel Tropes et en essayant de retirer les mots parasites, on relève que les verbes les plus fréquents sont remercier, lire et aimer, le substantif le plus utilisé est merci, et les adjectifs récurrents sont : grand, passionnant, bon, pédagogique, agréable, riche, excellent, formidable, magique, intéressant... Les apprenants saluent la qualité des cours et la diversité des thèmes abordés, et apprécient d'avoir découvert de nouvelles œuvres de fantasy via le cours et les discussions sur le forum. Les mêmes points reviennent quand l'équipe pédagogique questionne les apprenants dans les fils «Votre avis sur le MOOC nous intéresse " et "Ce que vous avez aimé ». On constate cependant que Tropes remarque un "style plutôt narratif» dans les fils spontanés et un "style plutôt 
argumentatif» dans les fils créés par l'équipe pédagogique : autrement dit, les topics spontanés font mieux ressentir l'expérience personnelle et le plaisir quand les topics de l'équipe pédagogique semblent inviter les apprenants à se montrer plus factuels.

Les réactions face au contenu du MOOC, bien que globalement positives, ne sont pas non plus sans critiques. Certains apprenants, par exemple, ont réagi de façon mitigée à l'évaluation par QCM: soit les questions leur semblaient formulées de manière ambiguë, soit ce type d'évaluation leur paraissait trop «facile». Mais la plupart des critiques portent sur le cours de la semaine 4 , consacré à la dark fantasy et à la romance paranormale et évoquant, entre autres, Le Fantôme de l'Opéra ou encore Buffy contre les vampires. Nous avons relevé, pour la première édition du MOOC, 13 fils dédiés à ce sujet, dont les titres sont plus ou moins explicites: "Grosse déconvenue ", "Jusque-là tout allait bien », "MOOC ou congrès pour érudits ». C'est surtout le vocabulaire du cours qui semble poser problème : il est jugé «trop littéraire » et inadapté au grand public auquel le MOOC s'adresse. Nous pouvons observer, par exemple, un message de Sevleveque, qui reconnait «un fond érudit, enrichissant, fouillé, débordant de références » et « des analyses poussées » mais qui déplore « une forme détestable, une propension à nous considérer comme des étudiants ayant suivi un parcours classique de fac (de lettres ?), avec des prérequis parfaitement assimilés (pourtant 'Ce cours est destiné au grand public et aux amateurs du genre', 'Il n'y a pas de prérequis' est-il indiqué dans la fiche de présentation du cours »).

Ces critiques ne font cependant pas l'unanimité et certains apprenants estiment au contraire, comme DavidHerve, que «[...] ce cours est de niveau universitaire, il est nécessaire d'employer un vocabulaire spécifique pour cerner exactement les concepts. Je trouve au contraire que de manière générale les enseignants font preuve de retenue quant à l'usage des termes techniques de l'analyse littéraire. Si vous voulez vraiment voir de l'analyse littéraire 'hardcore' je vous conseille de jeter un œil à FIGURES III de Gérard Genette. »

Nous remarquons, dans ces échanges, une opposition entre les apprenants qui se sentent limités par leur absence de maîtrise des codes disciplinaires, et ceux qui soit ne rencontrent pas cette difficulté soit qui y voient l'occasion d'enrichir leurs connaissances. Nous pouvons en déduire que les démarches des apprenants sont plus ou moins actives: certains espèrent absorber la connaissance simplement en visionnant (ou en écoutant) les cours quand d'autres lisent les pdf, recherchent, complètent, explorent. L'utilisateur nthim est un bon exemple de cet investissement accru : «[...] Selon moi l'intérêt de ces cours en ligne est d'aller au-delà de ce que l'on connaît ou croit connaître. Si parfois des efforts sont nécessaires pour bien comprendre le vocabulaire ou le choix des enseignants, alors faisons-le. Lire et relire la transcription PDF, explorer les bibliographies, consulter les documents annexes, vérifier, recouper et approfondir les informations, c'est cela être étudiant, c'est cela suivre un cours, ce n'est pas attendre que cela vous entre dans le cerveau, tout préparé. Si la formation se veut massive et ouverte elle n'en est pas pour autant un supermarché en ligne où l'on clique pour avoir le produit désiré. » Ce que relève ici cet utilisateur du forum est très intéressant, et on remarque en effet, dans les critiques adressées au cours sur la dark fantasy, presque une démarche de consommateur insatisfait - c'est ce qui ressort du message de Sevleveque, qui semble dénoncer une forme de publicité mensongère. 
29 Mais que les différents modules du cours soient appréciés ou critiqués, ils sont dans tous les cas une source inépuisable d'échanges et de débats entre les apprenants. Dans leur étude du forum d'un MOOC de formation d'enseignants, Charlotte Dejean et François Mangenot ont remarqué que «[...] les discussions ne donnent lieu à aucun débat. [...] Il s'agit plutôt d'abonder dans le sens d'un point de vue ou d'une proposition concrète présentée par un intervenant en le complétant ou le commentant, d'apporter des expériences personnelles $[. ..] \aleph^{13}$. C'est ici loin d'être le cas: les apprenants du MOOC fantasy ont beaucoup débattu au cours des trois éditions, se sont conseillés quantité de livres et ont abordé des sujets très variés: merveilleux scientifique, escapisme, adaptations cinématographiques ou télévisuelles, questions de traduction, etc.

30 Nous ne retiendrons ici que leur sujet de discussion privilégié : la taxinomie, qu'il s'agisse de la distinction entre fantasy et science-fiction, entre fantasy et fantastique, ou encore de la définition des différents sous-genres de la fantasy. Nous avons en effet relevé 47 fils qui abordent ces questions dans la première édition du MOOC, 24 dans la deuxième et 37 dans la troisième, soit 108 en tout, sachant que ce décompte exclut délibérément les topics « doublons » qui n'ont pas su générer de discussion suivie et ne comportent qu'un ou deux messages. Certaines œuvres reviennent de façon récurrente dans leurs interrogations: les apprenants se penchent ainsi sur la question de l'appartenance générique de Star Wars, de Dune de Frank Herbert, de La Romance de Ténébreuse de Marion Zimmer Bradley, ou encore, de façon plus générale, sur les œuvres complètes de Lovecraft, de Roald Dahl ou de Jules Verne. Les apprenants discutent les définitions du cours, questionnent l'appartenance des œuvres qu'ils connaissent à l'un ou l'autre des sous-genres, et cherchent également à définir euxmêmes les mouvements, anticipant ou prolongeant, pour certains, un des exercices de validation du MOOC, qui impliquait de rédiger une définition détaillée et complète de la fantasy en tant que genre. Certains sous-genres plus ciblés, comme le steampunk, la gaslight fantasy et la romance paranormale sont constamment débattus et redéfinis au fil des interactions. Dans la majorité des cas, les définitions des utilisateurs passent par la référence à de nombreux exemples, convoquant des œuvres plébiscitées et connues de tous afin d'amorcer de nouveaux mouvements définitoires. Les topics concernant ces opérations d'étiquetage des genres sont non seulement nombreux, mais ils sont également fertiles, et engendrent des débats étendus sur plusieurs messages, chacun donnant sa propre version de la définition discutée.

31 Pour autant, quand nous avons questionné les apprenants de la troisième édition du MOOC concernant l'influence de ces classifications sur leurs pratiques de lecture, les réponses ont, presque unanimement, nié l'importance de la taxinomie dans leur appréciation des œuvres littéraires. On lit, par exemple, sous la plume de Nazonfly: "Comme ma façon de découvrir des livres est assez aléatoire, les classements m'importent peu. Ou, plus exactement, les débats sur les classements m'importent peu : chacun a sa propre définition et il est assez vain de savoir si telle ou telle œuvre est de tel ou tel genre. » ou encore, sous celle de BenLal : "Les classifications en sous-genre ont peu d'importance pour moi en tant que lecteur/spectateur/joueur.»

32 Ce manque d'intérêt revendiqué pour la taxinomie avait déjà été relevé par Élodie Hommel dans sa thèse sur les lecteurs de science-fiction et de fantasy. En effet, elle y remarque que ses enquêtés ont tendance à rejeter les questions de classification. Les propos tenus par les lecteurs interrogés recoupent les témoignages des apprenants du 
MOOC : “'Moi j'ai jamais labellisé dans ma tête l'appartenance d'un roman à un genre' (Maxime), 'Enfin dans mon esprit, je suis pas tout le temps à cataloguer, ça, ça, ça. ..' (Ophélie), 'C'est compliqué de mettre des étiquettes' (Marina). » ${ }^{14}$

Ce rejet d'une taxinomie envisagée comme " posture savante $»^{15}$ rejoint tout à fait les critiques relevées plus tôt sur le cours de la semaine $4:$ une partie des enquêtés d'Élodie Hommel et des apprenants du MOOC semble rebutée par une certaine forme d'érudition. Cette posture est même assez surprenante dans le cas des apprenants et des enquêtés qui se définissent comme "littéraires. " $\mathrm{Si}$ on a vu que certains professeurs, documentalistes et bibliothécaires s'étaient justement inscrits au MOOC dans le but d'approfondir leurs connaissances professionnelles, certains, au contraire, dissocient la posture savante des "lectures plaisir." Ainsi, lors du MOOC 3, une apprenante, Liriel, écrit: "Je suis de formation littéraire et je comprends la raison d'être des classifications en bibliothèques et en librairies, mais en tant que lectrice, elle m'importe peu car je lis de tout. Je suis plus sensible au style de l'auteur et au 'réalisme' de l'histoire. » Élodie Hommel mentionne le cas d'une de ses enquêtées, Ophélie, professeur de français, qui «adopte un type de lecture savant et analytique face aux œuvres classiques étudiées en cours ou lues pour compléter ses connaissances, [mais] ne se pose pas ce genre de questions quand il s'agit d'un roman de science-fiction lu pour se détendre. " ${ }^{16}$ Élodie Hommel rapproche cette attitude des "pratiques culturelles dissonantes des individus de classes supérieures, et notamment des professeurs de lettres, mises en avant [par] Bernard Lahire ${ }^{17}[\ldots] \aleph^{18}$ : certains amateurs se définissant comme « littéraires » dissocieraient ainsi une posture analytique d'ordre professionnelle d'une posture de pur plaisir dans la pratique des lectures dites de divertissement.

Pour autant, Élodie Hommel remarque que ses enquêtés, tout comme les apprenants du MOOC, prennent plaisir à créer ce qu'elle appelle des "catégories profanes " ${ }^{19}$, à discuter des genres et sous-genres pour effectuer des distinctions et des rapprochements entre leurs différentes lectures. Ainsi, lors de la troisième édition du MOOC, Scifan écrit : «Personnellement la classification en sous-genres est un exercice que je réserve à ce MOOC. », secondé par Fabbygael : «[...] j'adore les débats que ces classifications engendrent, car elles sont un levier à la discussion assez simple mais toujours riche car il est plutôt rare de s'entendre sur la classification d'une œuvre. » $\mathrm{Si}$ la classification ne revêt donc pas une importance capitale lors de l'expérience de lecture, elle permet d'engendrer des discussions fertiles entre amateurs, et serait ainsi une stimulation puissante lors des débats sur le forum. Ranger, étiqueter et classer ne sont donc pas des mouvements intellectuels nécessaires à l'appréhension des œuvres, mais des prétextes à la discussion et au débat au sein d'un fandom constitué, d'une communauté d'amateurs partageant un référentiel commun.

Ainsi, les apprenants ont, lors des trois éditions du MOOC, échangé avec assiduité sur les forums. Mais leur investissement dans le MOOC ne s'est pas limité au plaisir de la discussion, puisque certains d'entre eux ont participé à une entreprise collaborative faisant ainsi pencher le MOOC fantasy du côté des MOOC de type c, pour " connectivistes ", dans lesquels, comme l'explique Pierre Lévy, " ce sont d'abord les relations et le partage de compétences des apprenants qui sont privilégiées. ${ }^{20}$ 


\section{Retransmission et création de savoirs}

Parmi les apprenants du MOOC Fantasy, certains ne se sont en effet pas contentés de regarder les vidéos puis de faire les exercices, mais se sont réellement investis dans la construction d'une communauté d'apprentissage en partageant leurs propres savoirs personnels ou en relayant la parole des professeurs. Comme l'écrit Denis Cristol, « Avec la possibilité de faire irruption sur un média tel qu'Internet, voire même de produire des idées et de les diffuser via un blog, l'amateur conquiert sa part d'indépendance. [...] Chacun est libre aujourd'hui de dépasser les barrages qui limitaient, et parfois censuraient, l'expression individuelle. L'expert et l'amateur sont à cet égard à égalité de clavier. ${ }^{21}$ Cette confusion entre apprenant et formateur est, par moments, tout à fait perceptible dans le MOOC Fantasy, et prend plusieurs formes.

Il est arrivé assez fréquemment, au cours des trois éditions du MOOC, que des apprenants soient en désaccord plus ou moins important avec le cours, et qu'ils ouvrent un topic pour critiquer ou nuancer le cours en question en s'appuyant sur leurs propres lectures. Nous n'évoquerons ici qu'un seul exemple : une apprenante critiquant un des éléments du cours d'Emmanuelle Poulain-Gautret, à savoir l'idée que les héros de fantasy seraient souvent porteurs de valeurs chevaleresques. L'apprenante cite plusieurs sources bibliographiques pour illustrer sa contre-argumentation : Conan ${ }^{22}$, ou encore Les Chroniques de la guerre de Lodoss ${ }^{23}$.

D'autres apprenants, sans montrer de désaccord avec le cours, ont souhaité l'enrichir en ouvrant des fils dédiés à des références culturelles de toutes sortes (romans, séries, films, jeux vidéo, groupes de métal, etc.) parfois commentées. Dans notre inventaire des fils, nous en avons compté, sur les trois éditions du MOOC, une trentaine qui ont été vraiment développés et suivis. Si le vocabulaire trop « littéraire » ou « jargonnant » est ainsi considéré comme une posture trop érudite, une érudition de l'accumulation, de la liste, semble au contraire plutôt valorisée.

Le MOOC fantasy a également vu émerger des apprenants particulièrement actifs et investis sur le forum, auxquels ont été attribués le statut de "Community Teaching Assistant ", ou TA. Ce statut permet d'accéder aux droits de modération des messages sur le forum, et confère également à ces apprenants une légitimité accrue pour répondre aux questions de leurs pairs. Il s'agit nettement des "personnes-ressources » dont parlent Dejean et Mangenot $^{24}$, ou encore des «individus-relais » mentionnés par Denis Cristol $^{25}$, qui se distinguent de leurs pairs par un investissement accru dans les activités du MOOC et qui deviennent des relais de la parole des formateurs. La hiérarchie qui s'établit entre apprenants et TA est véritablement, ici, d'ordre pédagogique: alors que Christian Le Bart remarque, dans son étude des fans des Beatles $^{26}$, que les hiérarchies entre amateurs, bien que jouant sur divers critères, se fondent sur un degré de proximité avec le groupe et sa musique ${ }^{27}$, et donc sur une question de «qui est le plus fan » ou de "qui est le meilleur fan »; nous observons ici que les TA s'identifient essentiellement à des étudiants attentifs, qui maîtrisent mieux que les autres le contenu du cours. Ils défendent une posture d'étudiant sérieux, et non de « meilleur fan de fantasy ». Ils répondent en effet majoritairement aux questions des apprenants en les renvoyant au texte du cours, en le citant ou le paraphrasant, pour éviter aux enseignants de répéter une même explication sur trop de fils; et relaient les questions des apprenants aux enseignants quand le cours ne contient pas les réponses suffisantes. 

démonologie »: un apprenant se pose la question de l'appartenance de l'œuvre de Lovecraft au genre fantastique ou à la fantasy, et la discussion, qui implique une TA nommé Djulaie, soulève la distinction entre l'anglais «fantastic» et le genre fantastique dans sa définition française. Par la suite, Djulaie ouvre un fil dédié à cette question, avec un intitulé clair, "le fantastique chez les anglais», pour poser directement cette question à l'équipe pédagogique et obtenir une réponse d'Anne Besson pour les autres apprenants.

41 Les évaluations par les pairs, très souvent pratiquées dans les MOOC, ont également contribué à cette confusion entre professionnels et amateurs en permettant à l'apprenant de faire une incursion temporaire dans le monde de l'enseignant. Dans le parcours de difficulté supérieur de la deuxième et de la troisième édition du MOOC, le «parcours mage », les apprenants peuvent choisir de devenir un «mage du savoir » ou un «mage de la création » et d'écrire soit un texte argumentatif soit un texte de fiction ou de réaliser un support visuel (que l'on peut rapprocher d'un fanart), lesquels sont ensuite évalués par les pairs. L'évaluation proposée est critériée et les évaluateurs peuvent détailler leur notation et inclure des appréciations. Cette transition de l'apprenant à l'évaluateur ne s'est pas faite sans difficulté, et les trois forums sont parsemés de fils questionnant l'évaluation par les pairs, les critères, la forme et la longueur du texte demandé, et surtout: pourquoi leur production a été mal notée et pourquoi ils ne sont pas devenus « mages. »

Bien que les apprenants aient fait cette expérience de l'évaluation par les pairs, le système hiérarchique avec professeur, TA et apprenant témoigne encore d'un modèle d'apprentissage traditionnel où la transmission de connaissance s'effectue de manière verticale. Mais cette configuration n'est pas la seule proposée par le MOOC fantasy, puisque d'autres modalités d'apprentissage impliquant les apprenants en tant que membres d'une communauté ont été mises en place, comme l'atelier de sous-titrage, auquel plusieurs apprenants et TA ont contribué pour permettre à leurs pairs malentendants de pouvoir regarder les vidéos. Mais l'activité qui a le mieux permis aux apprenants de se retrouver autour d'un projet commun a été l'élaboration du wiki. Celui-ci comprend en effet un lexique collaboratif, un répertoire des œuvres mentionnées qui a permis aux apprenants de réaliser un travail de folksonomie ${ }^{28}$ (ce qui constitue, pour Denis Cristol, «le premier pas d'une communauté d'apprentissage » ${ }^{29}$ ), ainsi que des histoires collaboratives.

ffet, certains des apprenants du MOOC sont aussi devenus auteurs de fantasy et ont écrit ensemble des textes de fiction. Dans la première édition du MOOC, ils se sont organisés sur le fil « Le coin des auteurs » et ont créé plusieurs groupements d'histoires tissant des liens entre elles, les séries A, B et C. Dans beaucoup de ces récits, les aventuriers parcourent le pays du MOOC en suivant la carte proposée par les formateurs : le pays d'Historia (semaine 1), le pays imaginaire (semaine 2), la région de Bannières puis les Marches du légendaire (semaine 3), Thanateron pour la semaine 4 et enfin Mediatika (semaine 5.) La série la plus prolifique a été la B : les auteurs de celle-ci se sont organisés sur un fil pour discuter au fur et à mesure de leur création - un fil qui compte tout de même 134 messages. Cette série fait intervenir le personnage de Roland créé par le TA Scifan et met en scène, par moments, les professeurs du MOOC comme personnages de l'histoire : 
«Le brouhaha s'éteignit doucement, lorsqu'une femme entra sur l'estrade et s'assit sur un fauteuil de cuir, elle se présentât [sic] dans le plus complet silence.

- Bonjour, je m'appelle Anne Besson, je suis maître de conférences habilitée à diriger les recherches à l'Université d'Artois, et spécialiste de fantasy.

Roland compris [sic] qu'elle était une Érudite, [...] elle parla de la galaxie Fantasy, de ses origines, de mondes qu'il avait visités, de mondes dont il connaissait le nom, de mondes qui lui étaient totalement inconnus, elle parla de conteurs, et d'Érudits, elle parla d'époques, et de médias. » pratique les savoirs acquis lors du MOOC en les intégrant à des aventures et ont mis en scène via la fiction leur propre processus d'apprentissage. On est donc bien, ici, dans une situation de knowledge building, c'est-à-dire de «[co-élaboration] des savoirs par engagement collectif autour d'un projet commun. ${ }^{30}$ " Les apprenants qui ont participé à ces projets ont formé une véritable communauté d'apprentissage avançant ensemble dans son exploration et sa construction des savoirs. Cet investissement intense n'est bien entendu pas seulement celui d'étudiants sérieux et impliqués, mais bien celui d'apprenants-fans, qui trouvent dans le MOOC un moyen de se rassembler autour d'une pratique de lecture-plaisir, d'une passion partagée. Christian Le Bart remarque, dans son étude des fans des Beatles parue en 2004, un même élan à la fois de sociabilité et de créativité au sein de la communauté : «Dans tous les cas, la rencontre suscite une sociabilité active. La passion peut désormais être dite, et avec elle toutes les directions dans lesquelles elle se déploie : collection de disques, de livres, d'objets, de photos, accords débutants ou confirmés sur une première guitare, désir d'imiter ou de composer... $»^{31}$. Les apprenants les plus assidus du MOOC fantasy sont indubitablement des fans qui expriment leur passion via toutes les possibilités offertes par le support, de l'échange entre pairs à l'écriture créative et, pour certains, au fanart. Pour ces apprenants, l'identité de fan ne se restreint pas à la seule expertise de l'objet, mais s'exprime aussi via une véritable entreprise de création collaborative qui dépasse la simple validation du MOOC.

\section{Conclusion}

46 Ainsi, le MOOC fantasy de l'université d'Artois a non seulement rassemblé un grand nombre d'inscrits et atteint un très bon taux de complétion, mais il a également permis l'émergence d'une communauté d'apprentissage. Les échanges entre pairs ont été fertiles et les projets collaboratifs ont permis à un bon groupe constitué d'apprenants de mettre en pratique les savoirs accumulés et de développer des compétences littéraires et rédactionnelles. Les apprenants n'ont pas seulement reçu le savoir dispensé, ils se sont également rassemblés autour de projets communs pour construire leurs propres apprentissages. Rien d'étonnant donc qu'après cette aventure, les 
histoires collaboratives de la troisième édition du MOOC se terminent par une scène de festin, contée par Scifan, qui rassemble apprenants, TA et professeurs et qui s'achève de la façon suivante: "Commence l'ultime festin, sans barde, présidé par les Érudites, Anne Besson, Isabelle Olivier, Emmanuelle Poulain-Gautret et Myriam White-Le Goff, toutes rendent hommage à leur collègue Isabelle-Rachel Casta ${ }^{32}$ cernée par les polyphonies insulaires. Enfin déchargé de sa mission de sauveteur en haute mer, Daniel Le Berre ${ }^{33}$ les accompagne. Les acclamations retentissent et les libations commencent. Le MOOC est fini.»

\section{BIBLIOGRAPHY}

Anne-Sophie Béliard, « Pseudos, avatars et bannières : la mise en scène des fans ", Terrains \& travaux, $\mathrm{n}^{\circ} 15$, septembre 2009, p. 191-212.

Philippe Carré, André Moisan et Daniel Poisson, L'autoformation : psychopédagogie, ingénierie, sociologie, Paris, France, Presses universitaires de France, 1997.

Bernadette Charlier, et Emmanuelle Annoot, La pédagogie universitaire à l'heure du numérique : questionnement et éclairage de la recherche, éds. Geneviève Lameul et Catherine Loisy, Louvain-laNeuve, De Boeck Supérieur, 2014.

Caroline Chemosit, John Rugutt et Joseph K. Rugutt, Fostering Sustained Learning Among Undergraduate Students: Emerging Research and Opportunities, Hershey, IGI Global, 2017.

Antoine Compagnon, « Moocs et vaches à lait », Le Débat, mai 2014, p. 170178.

Denis Cristol, Les communautés d'apprentissage : apprendre ensemble à l'ère numérique, Paris, ESF éditeur, 2016.

Charlotte Dejean, et François Mangenot, « Les interactions entre pairs dans un MOOC de formation d'enseignants », Ela. Études de linguistique appliquée, 2016, p. 419431.

Élodie Hommel, Lectures de science-fiction et fantasy : enquête sociologique sur les réceptions et appropriations des littératures de l'imaginaire, Thèse de doctorat, Université de Lyon, 2017.

Thierry Karsenti, «Les MOOC, révolution ou simple effet de mode? », Revue internationale des technologies en pédagogie universitaire, vol. 10 / 2, 2013, p. 622.

Christian Le Bart, «Stratégies identitaires de fans », Revue française de sociologie, vol. 45, 2004, p. 283-306.

Pierre Lévy, L'intelligence collective : pour une anthropologie du cyberspace, Paris, La Découverte, 1997.

Michel Marcoccia, «L'analyse conversationnelle des forums de discussion : questionnements méthodologiques », Les Carnets du Cediscor. Publication du Centre de recherches sur la didacticité des discours ordinaires, novembre 2004, p. 2337.

Yves Michaud, «L'Université de tous les savoirs à l'heure de Wikipédia, de Youtube et des Moocs », Esprit, novembre 2013, p. 96107. 
Nicolas Oliveri, et Paul Rasse, Apprendre en ligne : quel avenir pour le phénomène MOOC ? Paris, L'Harmattan, 2016.

Caroline Vincent, «Les débuts d'une nouvelle forme d'interactions pédagogiques : étude d'un Mooc fondé sur les réseaux sociaux », Ela. Études de linguistique appliquée, 2016, p. 399418.

\section{NOTES}

1. FUN MOOC [en ligne]. Page de présentation du MOOC « Fantasy, de l'Angleterre victorienne au Trône de fer », 2017 [consulté le 05/09/2018]. Disponible sur : https://www.fun-mooc.fr/courses/ course-v1:univartois+35001+session03/about

2. Tropes est un logiciel d'analyse sémantique créé par Pierre Molette et Agnès Landré en 1994, et utilisé depuis lors pour de nombreux travaux de recherche.

3. Caroline Chemosit, John Rugutt et K. Joseph Rugutt, Fostering Sustained Learning Among Undergraduate Students: Emerging Research and Opportunities, Hershey, IGI Global, 2017.

4. Antoine Compagnon, « Moocs et vaches à lait », Le Débat, mai 2014, p. 170-178.

5. Thierry Karsenti, «Les MOOC, révolution ou simple effet de mode ?», Revue internationale des technologies en pédagogie universitaire, vol. 10 / 2, 2013, p. 6-22.

6. Le taux de complétion des MOOC est effet estimé à seulement $5 \%$ (voir Nicolas Oliveri et Paul Rasse, Apprendre en ligne : quel avenir pour le phénomène MOOC ?, Paris, L'Harmattan, 2016).

7. Philippe Carré, André Moisan et Daniel Poisson, L'autoformation : psychopédagogie, ingénierie, sociologie, Paris, France, Presses universitaires de France, 1997.

8. Anne-Sophie Béliard, "Pseudos, avatars et bannières : la mise en scène des fans ", Terrains \& travaux, $\mathrm{n}^{\circ} 15$, septembre 2009, p. 191-212.

9. Nous n'avons pas accès à ces informations pour la première édition du MOOC, et nous nous servirons des statistiques établies par l'équipe pédagogique. Pour la troisième édition, sur 6183 profils recensés, 748 n'ont pas renseigné leur année de naissance, 683 n'ont pas indiqué leur genre, 669 n'ont pas mentionné leur niveau d'étude, 498 n'ont pas précisé leur pays, et 2581 ont laissé la rubrique « objectifs » vierge.

10. Denis Cristol, Les communautés d'apprentissage : apprendre ensemble à l'ère numérique, Paris, France, ESF éditeur, 2016.

11. Les pratiques culturelles des français [en ligne]. Synthèse de l'enquête de 2008 [consulté le 05/09/2018]. Disponible sur: http://www.pratiquesculturelles.culture.gouv.fr/doc/ 08synthese.pdf

12. Nicolas Oliveri et Paul Rasse, Apprendre en ligne : quel avenir pour le phénomène MOOC?, Paris, L'Harmattan, 2016.

13. Charlotte Dejean et François Mangenot, «Les interactions entre pairs dans un MOOC de formation d'enseignants », Ela. Études de linguistique appliquée, 2016, p. 419-431.

14. Élodie Hommel, Lectures de science-fiction et fantasy : enquête sociologique sur les réceptions et appropriations des littératures de l'imaginaire, Thèse de doctorat, Université de Lyon, 2017.

15. Ibidem.

16. Ibidem.

17. Bernard Lahire, La culture des individus, Dissonances culturelles et distinction de soi, Textes à l'appui, série

Laboratoire des sciences sociales, Paris, La Découverte, 2004.

18. Élodie Hommel, op. cit.

19. Ibidem.

20. Pierre Lévy, L'intelligence collective : pour une anthropologie du cyberspace, Paris, La Découverte, 1997. 
21. Denis Cristol, op. cit.

22. Robert E. Howard, Conan le cimmérien, traduit par Patrice Louinet et François Truchaud, Paris, Bragelonne, 2007.

23. Ryo Mizuno, Chroniques de la guerre de Lodoss, traduit par Christèle Dufraisse, Laurence Poyer et Yukio Reuter, Paris, Calmann-Lévy, coll. « fantasy », 2006-2009.

24. Charlotte Dejean et François Mangenot, op. cit.

25. Denis Cristol, op. cit.

26. Christian Le Bart, «Stratégies identitaires de fans ", Revue française de sociologie, vol. 45, 2004, p. 283-306.

27. «À l'intérieur du monde Beatles, des hiérarchies existent mais elles sont, pour cette raison, flottantes. Au fil des discussions, on glisse d'un monde à l'autre, l'excellent guitariste qui sait jouer tous les morceaux doit avouer qu'il ne connaît l'histoire du groupe que dans ses grandes lignes. Tantôt on affirme une position supérieure dans un même monde ("Je joue mieux que toi.", “J'ai vu plus de concerts que toi.", "J'ai tous les disques.", etc.), tantôt on affirme la supériorité d'un monde sur un autre (“Tu sais tout de McCartney, mais moi je l'ai vu en vrai. "). ", ibidem, p. 297-298.

28. Le terme "folksonomie », francisé à partir de l'anglais «folksonomy», désigne un travail d'indexation effectué de manière collaborative et spontanée.

29. Denis Cristol, op. cit.

30. Ibidem.

31. Christian Le Bart, op. cit.

32. Les « érudites » ici mentionnées sont les enseignantes de lettres de l'université d'Artois ayant élaboré le contenu du MOOC fantasy.

33. Daniel Le Berre est le professeur d'informatique de l'univers d'Artois ayant géré les différents aspects techniques du MOOC fantasy.

\section{ABSTRACTS}

Cet article s'intéresse aux apprenants du MOOC fantasy créé par l'université d'Artois sur la plateforme FUN (France Université Numérique) et à leurs pratiques de lecture et d'apprentissage. Si les apprenants recherchent en effet, en s'inscrivant au MOOC, à s'instruire sur un genre fictionnel qu'ils apprécient, dans une démarche de fans, certains sont entrés dans une dynamique d'apprentissage plus complexe qui confond l'amateur et le professionnel, l'étudiant et le formateur, pour aller vers une construction collective et plurielle des savoirs.

INDEX

Mots-clés: MOOC, apprenants, fantasy, lecteurs, savoir 


\section{AUTHOR}

\section{MARIE LUCIE BOUGON}

Marie Lucie Bougon est doctorante contractuelle à l'université d'Artois et prépare une thèse de Littérature comparée intitulée La réception de la fantasy en France de 1972 à nos jours : de l'importation à l'appropriation sous la direction d'Anne Besson. Elle est membre de l'association LPCM (Littératures Populaires et Culture Médiatique) et a contribué au projet LégiPop en tant que vacataire.

Publication :

«L'imaginaire apocalyptique et son réinvestissement dans Battlestar Galactica de Ronald D.

Moore : du piège des prophéties à la quête de liberté », Philosopher avec Battlestar Galactica, dirigé par Monica Michlin, TV/Series n¹1, juin 2017. 